\title{
Conocimiento y participación de los padres en el cuidado del menor de cinco años, Instituto Especializado de Salud
}

\author{
Nuth Igvna Mezarina Ticona, ${ }^{1, a, b}$
}

Mezarina TNI. Conocimiento y participación de los padres en el cuidado del menor de cinco años, Instituto Especializado de Salud. Cuid salud, ene-jun 2017; $3(1): 267-276$.

\section{RESUMEN}

Introducción: la hospitalización y la enfermedad son eventos estresantes para el niño y los padres; afectando el bienestar subjetivo, las relaciones interpersonales y el desarrollo del niño. Objetivo: determinar el nivel de conocimiento y la participación de los padres en el cuidado del menor de cinco años hospitalizado en el servicio de medicina del Instituto Especializado de Salud. Material y Métodos: enfoque cuantitativo, diseño exploratorio descriptivo; realizado en un hospital de III-2 nivel en Lima-Perú, que utilizó como instrumento un cuestionario y una guía de observación aplicado a 201 padres entre octubre-diciembre del 2016; los datos fueron analizados mediante estadígrafos. Resultados: los padres de los lactantes obtuvieron un nivel de conocimiento medio (48\%) y de los infantes alto $(40,6 \%)$. En el cuidado, se destacó la participación moderada de los padres en ambos grupos (52\% y 49,5\% respectivamente). Entre las actividades realizadas con mayor frecuencia destaca el lavado de manos y la permanencia nocturna del padre/madre $\mathrm{y}$, las menos frecuentes las educativas y/o lúdicas. Conclusiones: el conocimiento sobre el cuidado del menor fue de nivel medio en los padres de lactantes y alto en los de infantes, participando de forma moderada en el cuidado del niño/a hospitalizado.

Palabras clave: conocimiento, familia, cuidado del niño, cuidado del lactante, relaciones padre-hijo, hospitalización (Fuente: DeCS BIREME).
Mezarina TNI. Knowledge and participation of parents in the care of children under five, Specialized Health Institute. Cuid salud, ene-jun 2017; 3(1):267-276.

\begin{abstract}
Introduction: Hospitalization and illness are stressful events for the child and parents; affecting the subjective well-being, interpersonal relationships and child development. Objective: To determine the level of knowledge and participation of parents in the care of children under five years hospitalized in the medical service of the Specialized Health Institute. Material and Methods: Quantitative approach, applied type, descriptive exploratory design; carried out in a III-2 level hospital in Lima-Peru, which used as a questionnaire and observation guide applied to 201 parents between October and December of 2016; the data were analyzed using statisticians. Results: The parents of the infants had an average level of knowledge $(48 \%)$ and of the infants high $(40.6 \%)$. In care, the moderate participation of parents in both groups $(52 \%$ and $49.5 \%$, respectively) was noted. Among the most frequently performed activities, it is worth noting the handwashing and the nocturnal permanence of the father and the less frequently the educational and / or playful activities. Conclusions: The knowledge about the care of the child was of average level in the parents of infants and high in the infants, participating moderately in the care of the hospitalized child.
\end{abstract}

Keywords: Knowledge, family, child care, infant care, parent-child relationships, hospitalization (Source: MeSH NLM).

Centro Médico del Corazón de San Pablo y Bilbao, Huancayo-Perú.

Licenciada en Enfermería. 


\section{INTRODUCCIÓN}

La hospitalización y la enfermedad son eventos altamente estresantes para el niño y los padres. Desde la visión de la salud mental, la hospitalización puede afectar el bienestar subjetivo, las relaciones interpersonales y el desarrollo del niño, producto del cambio de ambiente, separación de los padres, familia y amigos. Lo que hace fundamental la presencia familiar en el hospital, ya que es fuente de protección y seguridad para el niño. ${ }^{1}$

Ambos procesos son estímulos estresantes para el niño(a), dado que desconoce sobre la enfermedad; especialmente si es muy pequeño, no comprende su dolor y no entiende por qué sus padres lo abandonan en un ambiente extraño, que lo priva de sus amigos(as), objetos y juguetes favoritos. Desencadenando reacciones variadas, desde lloriqueos hasta el mutismo absoluto, pudiendo rehuir el contacto personal o estar dependiente del profesional. ${ }^{2}$

Lizasoáin, ${ }^{3}$ citando a la National Association for the Welfare of Children in Hospital-NAWCH-1, describe que esta asociación fue creada para ofrecer una serie de objetivos en favor de los niños hospitalizados, considerando que los padres no deben ser separados de sus hijos y que los hospitales deben dar facilidades que promuevan este vínculo. Al respecto, en 1986 el Parlamento Europeo difundió a través del Diario oficial de las comunidades europeas, la Carta Europea de los Derechos de los Niños Hospitalizados, del cual se destaca el: ${ }^{4}$

Derecho del niño a estar acompañado de sus padres o de la persona que los sustituya el máximo tiempo posible durante su permanencia en el hospital. Los padres, no deben ser espectadores pasivos sino elementos activos en la vida hospitalaria, sin que esto signifique costos adicionales. El ejercicio de este derecho no debe perjudicar, ni obstaculizar la aplicación de los tratamientos a los que debe ser sometido el niño. p. 69.

Norma que busca facilitar el proceso de adaptación del niño y sus padres al nuevo ambiente, logrando así mejorar la calidad de vida en el centro hospitalario. ${ }^{3}$ Dicho manifiesto ha promovido, en varios países del mundo, la creación de programas que ayudan a cumplir este derecho a través de leyes, como en el caso del Perú.

Aunque los padres no siempre están preparados para integrarse al servicio clínico, sobre todo cuando su hijo se hospitaliza por primera vez, debido a la angustia, desesperación, sentimientos de culpa, opiniones de otros $\mathrm{y}$, el escaso conocimiento sobre cómo cuidarlo; situación que produce a su vez entorpecimiento de su participación. Siendo necesario incluir programas o protocolos de acogida que promuevan la participación de los padres. ${ }^{5}$

La participación de la familia en el ambiente hospitalario busca promover un cuidado integral y entorno terapéutico óptimo. ${ }^{6}$ Contexto dónde la enfermera tiene un papel fundamental y determinante en el dinamismo de la acción al permitir la participación de los padres en el cuidado $;^{7,8}$ destacando que dentro de la diversidad de políticas de visitas la decisión final la toman las enfermeras, quienes modifican las reglas bien para flexibilizarlas o para cumplirlas al pie de la letra. ${ }^{9}$

Diversos estudios demuestran que la participación de los padres en el cuidado de los hijos disminuye los días de hospitalización, reduce las infecciones cruzadas, ${ }^{10,11}$ acelera el proceso de recuperación, ${ }^{10}$ reduce la tasa de ingreso y favorece las conductas y el aprendizaje de las madres; ${ }^{11}$ además de disminuir la letalidad en los lactantes. ${ }^{11}$ En el Perú poco se sabe sobre el efecto de estos programas en los hospitales, siendo el Instituto Nacional de Salud del Niño uno de los pioneros que favorece la visita de los padres y familiares, disponiendo para esto de un protocolo de acogida que busca que el paciente y su familia tengan la información necesaria para satisfacer las necesidades físicas, psicológicas y espirituales del niño hospitalizado. $^{12}$

Por lo expuesto, este estudio tiene como objetivo identificar el nivel de conocimiento y la participación de los padres en el cuidado del menor de cinco años hospitalizado en el servicio de medicina del Instituto Nacional de Salud del Niño.

\section{MARCO TEÓRICO}

Según la teoría de Vygotsky, citado por Carrera y Mazzarella, ${ }^{13}$ tanto el desarrollo como el aprendizaje interactúan entre sí, siendo el aprendizaje un factor para el desarrollo, donde el hombre se caracteriza por ser más social que biológico.

Lucci $^{14}$ refiere que la teoría de Vygotsky está centrada en las funciones psicológicas del individuo, clasificadas en procesos elementales y procesos superiores. El proceso elemental está basado en el desarrollo biológico, presente en los niños y en los animales; se caracteriza por las acciones involuntarias o reflejas que surgen de manera inmediata y sufren el control del ambiente externo. Respecto al proceso superior, éste es de origen social y solo está presente en 
el hombre como especie, caracterizado por la intencionalidad de acciones y porque son medibles; siendo resultado de la interacción entre los factores biológicos y los factores culturales que evolucionan al pasar los años. De este modo, se entiende que las funciones psíquicas tienen su origen en lo sociocultural, resultado de la interacción del individuo con su contexto cultural y social.

Otra teoría que fundamenta la comprensión de la etapa de desarrollo del infante es la de Erikson. De las 8 etapas vitales que prepone Erikson en el estudio se tomaron las tres primeras que corresponden a la infancia. Bordignon ${ }^{15}$ refiere que al ir superando cada etapa se desarrolla de conciencia y la personalidad en los individuos.

Sobre la confianza versus desconfianza-esperanza (cero a 18 meses), ésta es la etapa psicosexual del niño y comprende la asimilación de los patrones somáticos, mentales y sociales por el sistema sensorio motor, oral y respiratorio, mediante los cuales el niño aprende a recibir y aceptar lo que le es dado para conseguir ser donante. En esta etapa es importante que los padres, especialmente la madre, le ofrezca seguridad y satisfacción emocional, fortaleciendo el vínculo de confianza que será determinante a lo largo de toda su vida. $^{15}$

La autonomía versus vergüenza y duda (18 meses a 3 años), esta etapa comprende el periodo de maduración muscular, aprendizaje de la autonomía física, aprendizaje higiénico (control de esfínteres) y aprendizaje de la verbalización. Un excesivo sentimiento de autoconfianza y la pérdida del autocontrol en estos procesos pueden provocar la vergüenza y la duda. El equilibrio de estas fuerzas es importante para la formación de la consciencia moral, del sentido de justicia, de la ley y del orden; además de un equilibrio entre las experiencias de amor $\mathrm{u}$ odio, cooperación o aislamiento, autonomía o heteronomía; entre otros. Cumpliendo los padres un rol fundamental para el ejercicio de la autonomía y de la autoexpresión para la superación de la vergüenza, de la duda y del legalismo en la formación del deseo, de la ley y el orden. $^{15}$

Finalmente, la iniciativa versus culpa ( 3 a 5 años), esta etapa comprende el descubrimiento y aprendizaje sexual (masculino y femenino), una mayor capacidad locomotora y el perfeccionamiento del lenguaje. Estas capacidades predisponen al niño para iniciarse en la realidad o en la fantasía, en la identidad de género y sus respectivas funciones sociales y el complejo de Edipo, en el aprendizaje cognitivo y el afectivo. La fuerza distónica en esta etapa provoca sentimientos de culpa que nace del fracaso en los diversos aprendizajes y el miedo de enfrentarse a los otros en estas habilidades. El justo equilibrio entre la iniciativa y la culpa y el miedo es significativo para la formación de la consciencia moral, a partir de los principios y valores internalizados en los procesos de aprendizaje, en la iniciación del aprendizaje escolar, de la inserción social, a través de los prototipos ideales representados por sus padres, adultos significativos y la sociedad. ${ }^{15}$

También fue considerado la teoría de los cuidados de Kristy Swanson, quien considera al cuidado como una forma educativa de relacionarse con un ser apreciado hacia el que se siente un compromiso y una responsabilidad personal. Swanson ${ }^{16}$ compone su teoría de cinco procesos entre ellos: el conocer, definido como la lucha constante de querer entender algún evento, el estar con, centrado en la parte afectiva del usuario; el hacer, que engloba todas aquellas actividades que se han de realizar por el usuario resaltando que además del personal de salud, la familia también puede participar en el cuidado; el permitir, buscando como único fin el crecimiento de la familia para mejorar el autocuidado a través de la entrega de herramientas (conocimiento y/o materiales) y, por último, el de mantener las creencias, entendida como la de sostener una actitud optimista pero realista sobre los hechos.

\section{MATERIALES Y MÉTODOS}

El estudio fue de enfoque cuantitativo, método no experimental y diseño exploratorio descriptivo; realizado en el Instituto Nacional de Salud del Niño, entidad clasificada como nivel III-2, localizada en el distrito de Breña-Lima; específicamente en el servicio de medicina, dividido en $\mathrm{A}, \mathrm{B}, \mathrm{C}, \mathrm{D}$; eligiéndose los servicios $\mathrm{B}$ y $\mathrm{C}$ por ser áreas de infantes.

La población fue conformada por los padres de familia de niños(as) hospitalizados, cuya edad oscilaba entre los seis meses a cinco años. Para determinar el tamaño muestral se tomó como referencia los niños hospitalizados en el IV trimestre 2015 ( $\mathrm{N}=420)$ utilizando la fórmula para poblaciones finitas de estudios descriptivos ${ }^{17}$, considerando como criterios de inclusión: al padre/madre del niño(a) $\leq 5$ años, un tiempo de hospitalización mayor de 3 días; entre los de exclusión: padre/madre de niños(as) con enfermedades crónicas, en condición de iletrados, con más de una hospitalización del niño(a).

Para el tamaño muestral se consideró una confiabilidad del 95\%, con una proporción desconocida del evento esperado y un error de $5 \%$. De esta manera, 
la muestra fue conformada por 201 padres, elegidos mediante muestreo no probabilístico por conveniencia.

En la recolección de datos se utilizó la técnica de observación y la encuesta. El instrumento fue un cuestionario y una guía de observación elaborados con auxilio de la bibliografía. El cuestionario está basada en las etapas del desarrollo humano: Lactante ( 6 meses a 1 año 11 meses 29 días) e infante (2 años a 5 años); ambos contenían tres partes con 50 ítems, redactados en leguaje sencillo, comprensible y concreto: Datos generales (10 ítems); Conocimiento (20 ítems); Participación (20 ítems). Las respuestas para el variable conocimiento fueron dicotómicas y para la variable de participación una escala de graduación: siempre (4pts), casi siempre (3pts), a veces (2pts), casi nunca (1pt), nunca (cero pts).

La guía de observación estuvo orientada a caracterizar las actividades que realizan los padres como parte del cuidado según grupo etario (20 ítems). Ambos instrumentos fueron sometidos a juicio de expertos conformado por seis especialistas, entre profesionales pediatras (enfermeras y médicos), estadista y pedagogo. Las sugerencias se centraron en el cuestionario, invirtiéndose algunos ítems de (+) a (-) y reemplazando dos, el valor final de este proceso para el cuestionario fue de $p=0,004$ y para la guía de $p=0,003$.

Luego, el cuestionario se sometió a prueba piloto en el área de estudio con la participación de 40 padres. Los resultados de la variable conocimiento fueron analizados con el test de Kuder Richardson y la de participación con Alfa de Cronbach. El valor alcanzado en conocimiento fue de $p=0,80$ en ambos grupos etarios. En participación, el valor fue de $p=0,704$ en los lactantes y de $p=0,802$ en los infantes.

La definición de punto de corte para cada variable se realizó mediante escala de estaninos. El conocimiento en ambos grupos se clasificó en: Alto (18 a 20pts), Medio (15 a 17pts) y bajo (8 a 14pts). En participación, en los lactantes fue: Alta (66 a 80pts), Moderada (52 a 65pts) y Baja (36 a 51pts) y; en los infantes: Alta (68 a 80pts), Moderada (55 a 67pts) y Baja (42 a 80pts).

El conocimiento fue definido como la suma de ideas y nociones que tienen los padres sobre el cuidado al niño(a) hospitalizado, adquiridos mediante la experiencia y las enseñanzas del personal de salud $u$ otras personas significativas. Respecto a la participación, comprendía el desarrollo de actividades por parte de los padres en beneficio del niño(a) satisfaciendo sus necesidades biológicas, emocionales y cognitivas, además de colaborar en la terapéutica conforme lo indicado por el personal de salud.

Para recolectar los datos, inicialmente se cumplió con las coordinaciones administrativas; recolectándose los datos de octubre-diciembre 2016, previa coordinación con los jefes de servicio, además que los cuestionarios fueron aplicados por la misma investigadora. El contacto con los padres fue en el horario diurno en los respectivos servicios, explicándoles el objetivo del estudio; una vez obtenido su consentimiento verbal se hacía entrega de la hoja de consentimiento informado para su firma y luego el cuestionario. Las dudas o preguntas fueron absueltas de manera oportuna evitando inducir las posibles respuestas.

Respecto a la guía de observación, ésta fue aplicada en diferente momento identificando las actividades de cuidado que realizaban los padres con los niños(as), permaneciendo atentos durante aproximadamente seis horas, intercalando los servicios B y C entre días y turno mañana o tarde, cautelando que la observación no interfiera en el comportamiento cotidiano de los padres y niños(as).

El tipo de análisis de datos fue descriptivo, inicialmente los datos fueron tabulados mediante códigos en el Programa Excel 2013; luego transportados al paquete estadístico Stastical Package for the Social Sciences-SPSS V23.0 para procesarlos con la estadística descriptiva (porcentajes y frecuencias).

El proyecto fue aprobado por comité de ética del Instituto Nacional de Salud del Niño, Oficio No. 08362016-CEI-INSN. En el estudio se respetaron los principios bioéticos (autonomía, justicia, no maleficencia, beneficencia), además de la dignidad y el respeto a la persona, basados en los códigos internacionales y nacionales.

\section{RESULTADOS}

En los datos sociodemográficos, la edad media de los padres era 25 años, en su mayoría adultos jóvenes $(33,8 \%)$ y un importante $10,4 \%$ (21) era adolescente. Por otro lado, la madre era quien tenía mayor participación en el cuidado del menor (83,6\%/168); respecto al estado civil, el $60,7 \%$ de los padres estaba en situación de conviviente y $20,4 \%$ era soltero/a, con una media de 11 años de escolaridad, aunque un 21,9\% tenía estudios superiores no culminados. 
Sobre el número de hijos, el 47,8\% de los padres tenía un solo hijo(a), en situación laboral la mayoría reportó ser ama de casa $(63,7 \%)$ y un 4,5\% (9) estudiaba. Sobre si el padre o madre había recibido educación o fue orientado sobre los cuidados al hijo hospitalizado, el 75,1\% refirió que sí. Respecto a la edad del menor hospitalizado, el 50,2\% tenía entre 2-5 años de edad; identificándose que el 76,6\% de niños(as) permaneció hospitalizado de 3-15 días y solo un 5\% tenía más de 30 días. A seguir se muestran los resultados del estudio relacionados con las variables.

Gráfico 1: Nivel de conocimientos de los padres sobre el cuidado de los menores de cinco años, Instituto Nacional de Salud del Niño. Octubre-diciembre 2016

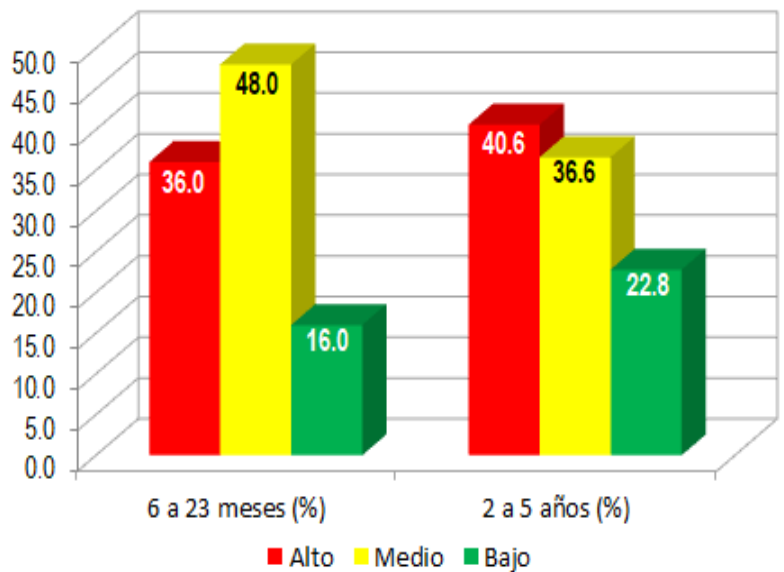

El gráfico 1 muestra que los padres de los lactantes de 6 meses a 23 meses tenían un conocimiento medio (48\%/48), mientras que los padres de los niños/as de 2-5 años destacó el conocimiento alto $(40,6 \% / 41)$.

Gráfico 2: Participación de los padres en el cuidado de los menores de cinco años, Instituto Nacional de Salud del Niño. Octubre-diciembre 2016.

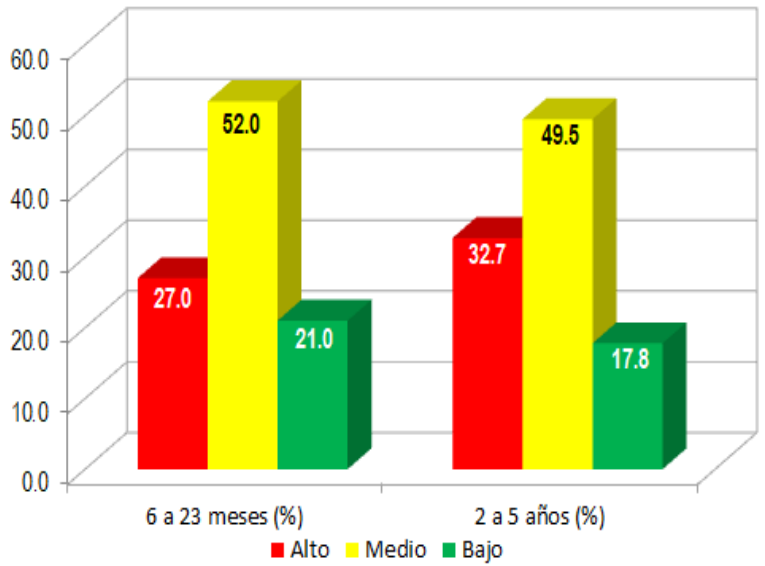

El gráfico 2 muestra que los padres de los niños(as) participan de manera moderada en el cuidado del niño/a hospitalizado con un 52\%(52) los padres de los lactantes y un $49,5 \%$ (50) los padres de los infantes, éstos últimos mostraron mayor habilidad en el cuidado del menor.

Tabla 1: Actividades que realizan los padres del lactante, Instituto Nacional de Salud del Niño. Octubre-Diciembre 2016.

\begin{tabular}{|c|c|c|c|c|c|c|}
\hline \multirow[b]{2}{*}{ Actividades realizadas por los padres } & \multicolumn{2}{|c|}{ NO } & \multicolumn{2}{|c|}{ A VECES \% } & \multicolumn{2}{|c|}{ SI } \\
\hline & $\mathrm{f}$ & $\%$ & $\mathrm{f}$ & $\%$ & $\mathrm{f}$ & $\%$ \\
\hline 1. Da de lactar. & 22 & 22 & 20 & 20 & 58 & 58 \\
\hline 2. Colabora en dar medicamentos. & 19 & 19 & 14 & 14 & 67 & 67 \\
\hline 3. Lava su manos antes y después de estar en contacto con el niño & 2 & 2 & 2 & 2 & 96 & 96 \\
\hline 4. Lava las manos de su niño(a) & 7 & 7 & 4 & 4 & 89 & 89 \\
\hline 5. Participa en el baño de su niño(a) & 4 & 4 & 4 & 4 & 92 & 92 \\
\hline 6. Cambia el pañal de su niño(a) & 8 & 8 & 11 & 11 & 81 & 81 \\
\hline 7. Aplica cremas para evitar las escaldaduras. & 8 & 8 & 10 & 10 & 82 & 82 \\
\hline 8. Colabora en la toma de temperatura. & 3 & 3 & 15 & 15 & 82 & 82 \\
\hline 9. Coloca paños húmedos en la frente, axilas, abdomen de su niño(a). & 13 & 13 & 13 & 13 & 74 & 74 \\
\hline 10. Lleva su juguete favorito. & 16 & 16 & 10 & 10 & 74 & 74 \\
\hline 11. Permanece por las noches. & 10 & 10 & 6 & 6 & 84 & 84 \\
\hline 12. Muestra cariño (coge de la mano, cabeza, habla amorosamente, entre otras). & 9 & 9 & 10 & 10 & 81 & 81 \\
\hline 13. Está presente durante la colocación del tratamiento endovenoso. & 16 & 16 & 18 & 18 & 66 & 66 \\
\hline 14. Cuenta cuentos. & 26 & 26 & 12 & 12 & 62 & 62 \\
\hline 15. Escucha música. & 46 & 46 & 12 & 12 & 42 & 42 \\
\hline 16. Tiene figuras para pintar & 63 & 63 & 11 & 11 & 26 & 26 \\
\hline 17. Garabatea, pinta, corta con su niño(a) & 51 & 51 & 13 & 13 & 36 & 36 \\
\hline 18. Sale a caminar con su niño(a) por los ambientes. & 26 & 26 & 13 & 13 & 61 & 61 \\
\hline 19. Ayuda a vocalizar palabras simples & 8 & 8 & 31 & 31 & 61 & 61 \\
\hline 20. La familia visita al niño(a). & 9 & 9 & 8 & 8 & 83 & 83 \\
\hline
\end{tabular}


La tabla 1 muestra que; un 96\%(96) de padres lavaba sus manos antes y después de tener contacto con su niño (ítem 3), 92\%(92) participaba del baño diario (ítem 5), 84\%(84) permanecería al lado del niño por las noches (ítems 11). Por otro lado, las actividades que realizan con menor frecuencia fueron las educativas, siendo que un $74 \%(74)$ de padres no llevaba figuras para que su niño(a) coloree (ítem 16), 51\% no lo incentivaba a escribir, garabatear o cortar (ítem 17) y $46 \%$ no escuchaba música con sus hijos (ítem 15).

Tabla 2: Actividades que realizan los padres de infantes, Instituto Nacional de Salud del Niño. Octubre-Diciembre 2016.

\begin{tabular}{|c|c|c|c|c|c|c|}
\hline \multirow[b]{2}{*}{ Actividades realizadas por los padres } & \multicolumn{2}{|c|}{ NO } & \multicolumn{2}{|c|}{ A VECES } & \multicolumn{2}{|c|}{ SI } \\
\hline & $f$ & $\%$ & $\mathrm{f}$ & $\%$ & $\mathrm{f}$ & $\%$ \\
\hline 1. Da de comer en diferentes horarios & 6 & 5,9 & 32 & 31,7 & 63 & 62,4 \\
\hline 2. Colabora en dar medicamentos. & 5 & 5,0 & 17 & 16,8 & 79 & 78,2 \\
\hline 3. lava sus manos antes y después de estar con su niño. & 2 & 2,0 & 25 & 24,8 & 74 & 73,2 \\
\hline 4. Lava las manos de su niño. & 6 & 5,9 & 9 & 8,9 & 86 & 85,2 \\
\hline 5. Participa en el baño diario. & 3 & 3,0 & 7 & 6,9 & 91 & 90,1 \\
\hline 6. Está pendiente de sus necesidades: orinar o defecar. & 2 & 2,0 & 11 & 10,9 & 88 & 87,1 \\
\hline 7. Mantiene seca la ropa de su niño(a). & 6 & 5,9 & 1 & 1,0 & 94 & 93,1 \\
\hline 8. Colabora en la toma de temperatura de su niño(a). & 2 & 2,0 & 11 & 10,9 & 88 & 87,1 \\
\hline 9. Coloca paños húmedos en la frente, axilas, abdomen de su niño(a). & 13 & 12,9 & 9 & 8,9 & 79 & 78,2 \\
\hline 10. Ha llevado el juguete de preferencia de su niño(a). & 3 & 3,0 & 2 & 2,0 & 96 & 95,0 \\
\hline 11. Permanece al lado de su niño(a) en las noches. & 0 & 0,0 & 4 & 4,0 & 97 & 96,0 \\
\hline 12. La relación con su niño es cariñosa. & 2 & 2,0 & 17 & 16,8 & 82 & 81,2 \\
\hline 13. Está presente durante el tratamiento endovenoso. & 9 & 8,9 & 25 & 24,8 & 67 & 66,3 \\
\hline 14. Cuenta cuentos a su niño(a) o escucha música. & 16 & 15,8 & 32 & 31,7 & 53 & 52,5 \\
\hline 15. Desarrolla pupileras/ juegos de palabras con su niño(a) & 40 & 39,6 & 27 & 26,7 & 34 & 33,7 \\
\hline 16. Sale a caminar con por los pasadizos. & 49 & 48,5 & 22 & 21,8 & 30 & 29,7 \\
\hline 17. Juega con su niño(a): ludo, ajedrez monopolio o usa su celular. & 63 & 62,4 & 20 & 19,8 & 18 & 17,8 \\
\hline 18. Permite que su niño(a) juegue con los niños del servicio. & 35 & 34,7 & 27 & 26,7 & 39 & 38,6 \\
\hline 19. El niño(a) recibe visita de sus familiares & 2 & 2,0 & 7 & 6,9 & 92 & 91,1 \\
\hline 20. Da tareas a su niño(a) como: pintar, rayar, cortar, seguir secuencias entre otros. & 15 & 14,9 & 23 & 22,8 & 63 & 62,3 \\
\hline
\end{tabular}

La tabla 2 muestra que el 96\% (97) de padres permanecía al lado de su niño(as) por la noche (ítem 11), $95 \%$ (96) llevaba consigo su juguete favorito (ítem 10), 93\% (94) secaba la ropa de su niño(a) (ítem 7). Asimismo, entre las actividades menos frecuentes el $62,4 \%$ (63) de padres no realizaban juegos con su niño (ítem 17), 39,6\% (40) no desarrollaba juegos de palabras (ítem 15) y 48\%(49) no solía caminar con su niño(a) por los ambientes de la hospitalización (ítem 16).

\section{DISCUSIÓN}

El estudio demostró que la madre es la principal responsable del cuidado del niño(a) hospitalizado, siendo una adulta joven y con un solo hijo, en su mayoría amas de casa y que vivían en situación de conviviente. $\mathrm{Su}$ permanencia en el hospital posiblemente se debía a que el padre se responsabiliza de la función económica de la familia, además que las normas del servicio no permiten la permanencia de ambos padres, excepto en el horario de visita.
Según el INEI $^{18}$, la edad en la cual las mujeres comienzan su vida reproductiva constituye uno de los factores demográficos determinantes en la fecundidad de una población. Por varios quinquenios, en el país, la edad mediana al nacimiento de la primera hija/o fue alrededor de 22 años, datos que en el estudio se muestran ligeramente elevados, aunque la media de 25 obtenida incluye también al padre.

Para Rojas, ${ }^{19}$ la edad ideal para ser madre y asumir la responsabilidad del hogar es a partir de 25-35 años en ambos sexos, etapa de la vida donde se asume con madurez la búsqueda del bienestar en la familia. En el estudio un $28 \%$ de los padres estaba en la edad adulta madura (mayor de 37 años); esta etapa se caracteriza por la madurez psicoemocional y sexual, lo que permite asumir el cuidado con responsabilidad y mayor adaptación emocional frente al proceso de enfermedad del niño(a) hospitalizado(a). ${ }^{19}$

La media de escolaridad de 11 años de los padres supone que estaban en mejores condiciones para 
comprender las orientaciones recibidas en el hospital del profesional de salud, pudiendo responder favorablemente en el cuidado del menor. Aunque como se ve, no están estimulando de modo continuo su desarrollo psicomotriz e intelectual (tabla 1 y 2).

Santander ${ }^{20}$ considera que tener un buen nivel de instrucción ayuda y mejora la participación de las madres en el cuidado de sus hijos(as). Aunque en el estudio los padres de los niños de 2-5 años parecen tener una mayor preparación en el cuidado (gráfico 1). Dato que podría fundamentarse en la madurez intelectual y emocional de los padres por la mayor edad, como también en la experiencia de estar cuidando del hijo mayor.

Dentro de los procesos básicos de Swanson, ${ }^{16}$ se resalta el proceso posibilitar, que se refiere a la de ofrecer herramientas al usuario/familia para incrementar sus conocimiento en base a la información que necesita o, apoyándolo con materiales; esto ciertamente con el fin de mejorar la participación de la familia durante la hospitalización.

Al respecto, el Instituto Nacional de Salud del Niño cuenta con una "Directiva Administrativa para la implementación de la visita de los padres y familiares", ${ }^{12}$ que ofrece información básica para favorecer la mejor adaptación del niño y su familia a la hospitalización, tratando de dar una respuesta integrada a las necesidades del niño(a) respecto a su bienestar físico, psicológico y social.

La participación de los padres es de suma importancia porque ayuda a los niños(as) y a los responsables del cuidado a adaptarse a la hospitalización, permitiendo que los padres apliquen lo aprendido en la orientación que reciben durante la hospitalización. En los resultados, el tipo de participación de los padres en el cuidado de lactantes e infantes fue moderada (gráfico 2).

Aunque no fue objetivo del estudio, parece que el nivel de conocimiento de los padres no necesariamente es determinante para participar más activamente en el cuidado del menor. Sin embargo, se identificó que los padres de los infantes tenían una alta participación, lo que podría interpretarse por la mayor edad de los niños y la experiencia ganada en ese recorrido.

Para Santander, ${ }^{20}$ al estar las madres con sus hijos evitan sentirse tristes, sintiéndose más tranquilas al observar cómo sus hijos se recuperan de la enfermedad. Además, reconocen que aprenden nuevas cosas y, sobre todo, que su presencia permite que sus hijos estén protegidos, se alimenten adecuadamente, reciban mejor tratamiento y casi no lloren.

Swanson ${ }^{16}$ refiere que el proceso básico hacer por, promueve la participación del personal de salud y de la familia para satisfacer las necesidades básicas del niño(a) hospitalizado; siendo de gran significado en el desarrollo psicológico de los infantes.

Entre las actividades de cuidado que realizaban los padres con el lactante destaca el lavado de manos, tanto de sí mismos como del niño; acción que ayuda a reducir la propagación de enfermedades infecciosas (tabla 1). Al respecto, Barrera et al. ${ }^{21}$ reportan que la participación materna ayudó a reducir la frecuencia de infección intrahospitalaria. Normatividad que se evidencia también en la Directiva de visitas del área de estudio. ${ }^{12}$

Otra actividad realizada por los padres fue el baño diario del infante, reconociendo que este acto relaja y disminuye la carga de bacterias en su cuerpo, puesto que en el contexto hospitalario siempre existe elevada contaminación ambiental.

Por otro lado, el $84 \%$ de padres permanecía al lado de su hijo(a) durante las noches; actividad que favorece el descanso de los niños, alcanzando las fases del sueño profundo que ayudaran en la regeneración de los tejidos, contribuyendo con la mejora de su estado de enfermedad. Partiendo de las etapas de desarrollo de Erickson $^{15}$, se asume que cada etapa es determinante para el perfeccionamiento de la personalidad del individuo y que ésta dependerá de la edad de desarrollo. Siendo significativa la presencia de los padres durante la hospitalización para favorecer la evolución normal, tal como lo afirma Erickson.

Entre las actividades menos frecuentes destacan las educativas y/o lúdicas; las cuáles se desarrollan de forma deficiente con posible impacto negativo en los niños, pues al no estimular su parte cognitiva y sus habilidades sensorio-motoras, éstas se debilitan pudiendo hasta regresionar dichas funciones en los lactantes.

En los infantes, las actividades realizadas con menos frecuencia por los padres involucran el acompañamiento durante la noche (tabla 2), además de no llevar al hospital su juguete favorito, no promover juegos que estimulen su desarrollo cognitivo, entre otros. Lo que justamente llama la atención, dado que los contenidos educativos deben seguir desarrollándose durante la hospitalización, sea mediante actividades lúdicas o, con actividades educativas tradicionales, por sus implicancias directas en el aprendizaje escolar. ${ }^{19}$ 
Las actividades lúdicas son reconocidas como una forma de disminuir la carga emocional que lleva consigo la hospitalización de niños(as). Según López y Fernández, ${ }^{23}$ citando a Palomo, el aburrimiento prolongado hace que el niño se entristezca y acabe adoptando actitudes pasivas e indiferentes a las actividades que le causaban alegría o bienestar, siendo que el juego en el hospital proporciona confianza y bienestar.

Todas las actividades que realice el niño durante la hospitalización evitarán la pérdida del hábito intelectual, además de compensar sus lagunas a nivel del aprendizaje evitando así el retraso escolar, favoreciendo la readaptación del niño al mundo educativo. ${ }^{23}$

Acciones que también deben ser consideradas dentro de la directiva de visitas de los padres y familiares, ${ }^{12}$ ya que los niños(as) están en pleno desarrollo biopsicosocial y por lo tanto, el cuidado debe cubrir todos los aspectos del ser humano, en una visión holística e integral, en la perspectiva de ofrecer un cuidado seguro (reduciendo las infecciones cruzadas) y eficaz, traducido como sanador logrando el mayor bienestar físico y emocional $\mathrm{y}$, motivador, permitiendo que los procesos neuronales e intelectuales sigan el curso de su máximo desarrollo cognitivo.

\section{Correspondencia:}

Nuth Igvna Mezarina Ticona

Correo electrónico: nuthmt20395@ hotmail.com

\section{REFERENCIAS BIBLIOGRÁFICAS}

1. Quirino DD, Collet N, Neves A. Hospitalización infantil: concepciones de la enfermería acerca de la madre acompañante. Rev Gaúcha Enferm, Brasil [serie de internet]. Jun 2010 [citado 03 may 2015]; 31(2): 300$306 . \quad$ Disponible en: http://www.scielo.br/scielo.php?script=sci_artte xt\&pid=S1983-14472010000200014

2. Rodríguez Bausa L. Características y déficit inherentes a la hospitalización infantil. Docencia e Investigación REVMT [serie de internet]. Dic 2009 [citado 03 may 2015]; 2: [cerca de 05 laudas]. Disponible en: http://www.uclm.es/varios/revistas/docenciaein vestigacion/numero2/luisrodriguez.asp
Entre las limitaciones del estudio se identifica el muestreo no probabilístico, en tal sentido los resultados solo son válidos para la población estudiada. Asimismo, puede considerarse también el cansancio de los padres por encontrarse todo el día en el hospital, lo que puede haber influido en el llenado del cuestionario generando distracción y/o automatismos en las respuestas. Por otro lado, durante el tiempo en que se desarrolló el estudio uno de los servicios del área de estudio tuvo un recorte en el ingreso de pacientes a hospitalización por remodelación en sus ambientes, hecho que redujo la afluencia de pacientes hospitalizados.

Es necesario que se continúen realizando estudios semejantes para observar mayor consistencia y especificidad de los instrumentos, ya que fueron elaborados partiendo de la literatura y de las teorías de desarrollo humano.

\section{Declaración de financiamiento y de conflictos de interés:}

El estudio fue financiado por la autora, declara no tener conflictos de interés.

\section{Contribución de auditoría:}

Mezarina TNI: Concepción y diseño del estudio, recolección de los datos, análisis e interpretación de los resultados y aprobación de la versión que será publicada

3. Lizasoáin RO. Los derechos del niño enfermo y hospitalizado: El derecho a la educación. Logros y perspectivas. ESE [serie de internet]. 2005. [citado 03 may 2015] 9: 189-201. Disponible en: http://www.dadun.unav.edu/bitstream/10171/89 23/1/NF.PDF

4. Parlamento Europeo. Carta europea de los niños hospitalizados. Bol Pediatr [serie de internet]. 1993 [citado 03 may 2015] 34: 69-71. Disponible en: http://www3.gobiernodecanarias.org/sanidad/sc s/content/35053fc9-3238-11e2-bbac-

2df7f25ac448/cartaeuropea.pdf 
5. Bosque AR. Participación familiar en una unidad de cuidados intensivos pediátricos. Opinión de enfermería. Rev Enferm CyL [serie de internet] 2015 [citado 04 may 2015]; 5 (2): 59-66. Disponible en: http://www.revistaenfermeriacyl.com/index.php /revistaenfermeriacyl/article/viewFile/88/79

6. Marco LM, Bermejillo EI, Pinedo NGF, Sarrate AI, Margall CMA, Asiain EMC. Creencias y actitudes de las enfermeras de cuidados intensivos sobre el efecto que la visita abierta produce en el paciente, familia y enfermeras. Rev. Enferm Intensiva [serie de internet]. 2000 [citado 6 may 2015] 11(3): [cerca de 11 laudas]. Disponible

en:

http://www.apps.elsevier.es/watermark/ctl_serv let?_f=10\&pident_articulo $=10017624 \&$ pident usuario $=0 \&$ pcontactid $=\&$ pident revista $=142 \& \mathrm{t}$ $\mathrm{y}=102 \&$ accion $=$ L\&origen $=$ zonadelectura $\& w e b$ $=w w w . e l s e v i e r . e s \& l a n=e s \&$ fichero $=142 \mathrm{v} 11 \mathrm{n} 3 \mathrm{a}$ 10017624pdf001.pdf

7. Vázquez CM, Eseverri AMC. El cuidado de las familias en las unidades de cuidados intensivos desde la perspectiva de Jean Watson. Enferm Intensiva [serie de internet]. Oct 2010 [citado 6 may 2015]; 21(4): 161-164. Disponible en: http://www.elsevier.es/es-revista-enfermeriaintensiva-142-articulo-el-cuidado-las-familiaslas-S1130239910000593

8. Torres PL, Morales AJM. Participación familiar en el cuidado del paciente crítico. Recomendaciones de la Sociedad Andaluza de Enfermería de Cuidados Críticos. Tempus vitalis [serie de internet]. 2004 [citado 6 may 2015]; 4(1). 18-25 Disponible en: http://www.tempusvitalis.es/TV_files/2004vol4 num1/5consenso41.pdf

9. Ramírez PCA, Parra VM. Percepción de los comportamientos del cuidado de enfermería en la unidad de cuidados intensivos. avan enferm [serie de internet]. 2011. [citado 6 may 2015]; 29(1):97-108. Disponible: http://www.scielo.org.co/pdf/aven/v29n1/v29n1 a10.pdf

10. Osorio A, Ferrari AM, Alonso R, Cardozo N. Hospitalización Conjunta Del Hijo Con Su Madre programa de madre participante. Bol Of Sanit Elec [serie de internet]. Jun 1979 [citado
13 may 2015]; 6(1): [cerca de 19 laudas]. Disponible en: http://www.hist.library.paho.org/Spanish/BOL/ v84n3p240.pdf

11.Barrera QF, Moraga MF, Escobar MS, Antilef HR. Participación de la madre y la familia en la atención del niño hospitalizado: Análisis histórico y visión de futuro. Rev.chil.pediatr [serie de internet] 2007. [citado 13 may 2016]; 78(1):164-8 [cerca de 05 laudas]. Disponible en:

http://www.scielo.cl/scielo.php?script=sci_artte xt\&pid=S0370-41062007000100012

12.Directiva administrativa Para la implementación de la visita de los padres y familiares. R.D. $\quad \mathrm{N}^{\mathrm{o}}$ 599-2015-INSN-DG. (2015).

13.Carrera B, Mazzarella C. Vygotsky: enfoque sociocultural. Educere,Venezuela [serie de Internet. 2001 [citado 10 may 2016]; 5(1): 41$44 . \quad$ Disponible en: http://www.redalyc.org/pdf/356/35601309.pdf

14.Lucci MA. La propuesta de Vygotsky: La psicología socio-histórica. Profesorado. Revista de Currículum y Formación del Profesorado [serie de internet]. 2006 [citado 19 jul 2017]; 10(2): 1-11. Disponible: http://www.ugr.es/ recfpro/rev102COL2.pdf

15.Bordignon NA. El desarrollo psicosocial de Erik Erikson. El diagrama epigenético del adulto. Revista Lasallista Investigación [serie de internet]. 2005 [citado 10 jun 2016]; 2(2): 50-63. Disponible en: http://www.redalyc.org/pdf/695/69520210.pdf

16.Swanson KM. Empirical development of a middle range theory of caring. Nursing Research [serie de internet]. May-jun 1991 [citado 10 ene 2015]; 40(3): 161-166. Disponible en: http://nursing.sites.unc.edu/files/2012/11/ccm3 032548.pdf

17.Martina CM, Gutiérrez VC, Alarcón ME, García CE, Whittembury VA, Terukina TR. Guía Teórica de Estadística Aplicada a las Ciencias de la Salud. Lima, Perú: UNMSM; 2014. 
18.Instituto Nacional de Estadística e Informática (INEI): Encuesta Demográfica y de Salud Familiar [internet]. Lima-Perú: INEI; 2014 [citado 10 feb 2017]. p.490. Disponible en: https://www.inei.gob.pe/media/MenuRecursivo/ publicaciones digitales/Est/Lib1211/pdf/Libro. pdf

19.Rojas CMR. Nivel de conocimiento y tipo de participación que tiene las madres durante la atención del niño hospitalizado en el Servicio de Clínica Pediátrica del HNERM [tesis de especialista]. [internet]. Lima-Perú: Universidad Nacional Mayor de San Marcos; 2005 [citado 10 nov 2015]. [cerca de 85 laudas]. Disponible en: http://sisbib.unmsm.edu.pe/bibvirtualdata/Tesis/ Salud/rojas_chm/rojas_cm.pdf

20.Santander MG. Programa de participación materna en el cuidado del lactante hospitalizado. Investigación y Educación en Enfermería [serie de internet]. Set 2001 [citado 03 may 2015]; 19(2): 52-56. Disponible en: https://www.aprendeenlinea.udea.edu.co/revista s/index.php/iee/article/download/16810/14562+ $\underline{\mathrm{cd}=1 \& \mathrm{hl}=\mathrm{es} \& \mathrm{ct}=\mathrm{clnk} \& \mathrm{gl}=\mathrm{pe}}$
21.Barrera QF, Sepúlveda BAM, Weber UC, Moraga MF, Escobar MS, Mejías JM. Participación materna en la atención del niño hospitalizado. Rev chil pediatr [serie de internet]. 1993 [citado 13 may 2016]; 64(3):164-168 [cerca 05 laudas]. Disponible en: http://www.scielo.cl/pdf/rcp/v64n3/art02.pdf

22.Hernández PE, Rabadán RJA. La hospitalización: un paréntesis en la vida del niño. Atención educativa en población infantil hospitalizada. Perspectiva Educacional [serie de internet]. Ene 2013 [citado 10 jun 2015]; 52(1): 167-168-181. Disponible en: https://www.dialnet.unirioja.es/servlet/articulo? codigo $=4174389$

23. López NI, Fernández CA. Hospitalización infantil y atención psico-educativa en contextos excepcionales de aprendizaje. Revista de Educación [serie de internet]. Sep-dic 2006 [citado 10 jun 2015]; 341: 553-574. Disponible en:

http://www.researchgate.net/publication/281327 73_Hospitalizacin_infantil_y_atencin_psicoducativa_en_contextos_excepcionales_de_apre ndizaje 